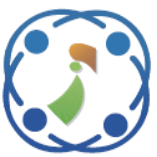

\title{
Energy-Efficient Cluster based Routing for Hybrid Wireless Sensor Networks using Adaptive Hybrid Cuckoo Search and Grey Wolf Optimization Algorithm
}

\author{
Manjunath Hanumanthappa Rangappa ${ }^{1 *}$ \\ Guruprakash Chirathahalli Dyamanna ${ }^{1}$ \\ ${ }^{I}$ Department of Computer Science and Engineering, Sri Siddartha University, Tumakuru, Karnataka, India \\ * Corresponding author's Email: manjunathdvg@gmail.com
}

\begin{abstract}
The combination of both static sensors and mobile sensors is called as Hybrid Wireless Sensor Network (HWSN). Static sensors monitor the environment and report events occurring in the sensing field. Mobile sensors are then dispatched to visit these event locations to conduct more advanced analysis. These sensor nodes have major energy constraints as they are small and their battery can't be replaced and collaborate together in order to gather, transmit/forward the sensed data to the base station. Consequently, data transmission is one of the biggest reasons for energy depletion in HWSN. Clustering is one of the most effective techniques for energy efficient data transmission in HWSN. This paper proposes an Adaptive Hybrid Cuckoo Search (AHCS) algorithm. AHCS improves the Lévy flight method and population evolution strategy of the cuckoo search algorithm, and introduced a mutation operation operator. Inspired by the idea of position update of Grey Wolf Optimization (GWO) algorithm, this paper introduces the inertia weight $w$ in the Lévy flight method of CS algorithm, and gives the new dynamic adjustment methods of parameters $\alpha$ and $\beta$ respectively. The proposed algorithm is evaluated in terms of response time, packet drop ratio, energy consumption, end to end delay and dead time. Furthermore, the performance of AHCS-GWO is evaluated with existing methods such as classical algorithms, Evolutionary Multipath Energy-Efficient Routing protocol (EMEER) and Yellow Saddle Goatfish Algorithm (YSGA). The residual energy of the AHCS-GWO method for 200 nodes is $0.57 \mathrm{~J}$, it is high when compared to the existing methods.
\end{abstract}

Keywords: Adaptive hybrid cuckoo search, Energy efficiency, Evolutionary multipath energy-efficient routing protocol, Grey wolf optimization, Hybrid wireless sensor network.

\section{Introduction}

For the past few years, hybrid process opens a new frontier of research in sensor networks with static and mobile nodes wireless sensor networks. Static sensors support environmental sensing and network communication. The general architecture of a Hybrid Wireless Sensor Networks (HWSN) including static and mobile nodes [1]. The static nodes include sink, normal nodes and partition heads; the mobile nodes are mobile relays. Mobile sensors are more resource-rich in sensing and computing capabilities and can move to particular locations to conduct more complicated missions such as repairing the network or providing in-depth analysis [2]. Introducing mobility to a WSN not only reduces its deployment and maintenance costs but also enhances its capability [3]. In recent years, sensor networks have become widely used in various applications, including military, agriculture, transportation, industry, and smart home applications. Typically, WSNs consist of hundreds or thousands of sensor nodes that are randomly and widely deployed. A sensor network provides a global view of a monitored area with local detection by each sensor [4]. However, there are a few inherent limitations in WSNs, such as low communication bandwidth, low storage capacity, and limited battery energy. Because of limited battery energy, it is very important how to collect the data from the sensors effectively and extend the lifetime of sensor network [5]. Most researchers use hybrid wireless sensor network including static and mobile nodes to raise the efficiency of data gathering. This kind of sensor network is divided 
into three types. In the first case, some sensors are the robots and can dynamically adjust the network topology when the holes occur in WSN [6]. In the second case, a mobile sink is utilized to collect data to prolong the lifetime of WSN. The energy consumption of nodes is not concentrated on several particular nodes, because the location of mobile sink is not fixed [7].

In the final case, the mobile nodes or mobile relays are assigned to collect data of sparse sensor network or partition network. The data flows are from the normal nodes to the sink. Two communication protocols are used in our method [8]. The first communication protocol uses ZigBee to communicate with static nodes. Another communication protocol uses Wi-Fi or ad hoc network to transmit data from the mobile relay to the sink. The data transmission is adjusted to detour around the region of hole while the hole developed [9].

And ensure that data can be delivered to the sink. However, the network finally generates partition due to the death of the last connecting node. The sink has to dispatch a mobile relay to collect data while the partition network will occur. The mobile relay goes to the location of near dead node to collect the data of sensors within the partition. Finally, it relays the data to the sink [10]. Therefore, the sink can collect all data of the entire sensor network successfully. Besides the transmission structure describe in this paper is a hybrid sensor network including static and mobile nodes.

In this paper, the main focus is on the $\mathrm{CH}$ selection issue and a AHCS-GWO based algorithm is presented called Whale optimization algorithm based clustering. The algorithm helps select the $\mathrm{CHs}$ among the sensor nodes efficiently taking into account the residual energy of the nodes. Algorithm is evaluated extensively to show its superiority over contemporary routing techniques.

The organization of the paper is given as follows: The contribution and literature review of this work are described in the section 2 and 3 respectively. The cluster based routing using AHCSGWO method is explained in the section 4. Section 5 provides the results and discussions of this AHCSGWO method. Finally conclusion is made in section 6.

\section{Contribution}

The main contributions of this study are as follows:
- To propose an adaptive hybrid cuckoo search with Grey Wolf Optimization to improve the energy efficiency.

- The Lévy flight method of the AHCS is improved. In the Lévy flight method of CS algorithm, this paper introduces the dynamic inertia weight $\mathrm{w}$, and gives the new adaptive adjustment methods of parameters $\alpha$ and $\beta$ respectively.

- The mutation operator is introduced and improved population evolution strategy of the AHCS is proposed.

- Compare the proposed AHCS-GWO with other improved algorithms in literature in the parameter optimization of the reducer and the proposed AHCS-GWO outperforms in quality of the solutions.

\section{Literature review}

Wang [11] developed a clustering algorithm with an improved artificial bee colony (ABC) algorithm to improve the energy efficiency and the lifespan of the network. Since the clusters used in WSNs perform more tasks, it consumes large amount of energy. Thus, the selection of optimal cluster heads becomes significant in WSN. Here, The $\mathrm{ABC}$ algorithm was used to implement the optimal clustering method by optimizing the fuzzy C-means clustering. The polling control mechanism based on busy/idle nodes was introduced into intracluster communication to minimize the energy consumption and improve the throughput of the network. However, the developed algorithm was able to utilize in only fixed networks.

Alghamdi [12] presented a route optimization algorithm to improve the QoS of the multi-hop wireless sensor network. The optimal path selection is important in WSN to improve the network performance. The other existing methods were considered only the selection of path with high bandwidth. But those methods were failed to analyze the other network parameters such as energy, distance and intensity of the traffic. The developed algorithm considered all those network parameters to provide better path with improved QoS. Since, all the adjacent nodes information was stored in the header of the optimum node at the next hop, the packet over head was minimized and the packet delivery ratio was improved. However, the optimum node selection takes a little more time at the beginning stage.

Nayyar and Singh [13] developed a novel energy efficient Ant Colony Optimization (ACO) based multipath routing protocol. The proposed ACO 
based routing protocol operates in three phases such as discovery of adjacent nodes through link knowledge, packet transmission through exponentially weighted moving average method and ACKR packet delivery for assuring end-to-end delivery. The proposed protocol improves the network performance in terms of packet overhead, energy efficiency, end to end delay, packet delivery and throughput. The ACK packet loss was occur during the transmission but it was not taking into consideration as long as the ACK pocket delivered to the source node at a reasonable amount of time delay.

Ezhilarasi and Krishnaveni [14] developed an evolutionary multipath energy-efficient routing protocol (EMEER) to improve the lifespan of the network. The optimal cluster head selection was done by introducing the Cuckoo Search Algorithm (CSA). The process of the optimization by using the CSA was composed of four parameters such as packet size, battery power, node location and hop distance. According to the four parameters, the CSA selected the optimal path for data transmission. The energy consumption of the network was minimized by adopting the routing scheme. However, the computational speed reduced for the maximum node count of 180 only.

Li [15] presented the novel Energy-Efficient Load Balancing Ant-based Routing Algorithm (EBAR) for WSNs. The power consumption of the sensor nodes were balanced by utilizing the improved pheromone trail update scheme and the pseudo- random route discovery algorithm. Here the optimal path selection process was done by introducing a greedy expected energy cost metric based efficient heuristic update algorithm. Since the energy based opportunistic broadcast scheme was used, the energy consumption of the network has been minimized. However, the path selection process in the EBAR requires maximum weight $\tau$.

$\mathrm{Xu}$ [16] developed novel energy efficient region source routing protocol (ER-SR) to prolong the network lifespan. In this ER-SR protocol, the source routing node was selected by introducing the distributed energy region algorithm. The source node was used to find the optimal routing path for an efficient data transmission. The energy consumption of the system was minimized by utilizing an effective distance-based ant colony optimization algorithm. Hence, the network lifespan was improved in the developed ER-SR protocol. However, the ER-SR protocol was able to use in the static networks only but not in the mobile networks.

Rodríguez, Del-Valle-Soto, and Velázquez [17] presented a novel energy-efficient clustering routing protocol based on Yellow Saddle Goatfish Algorithm (YSGA). The developed protocol was mainly introduced to strengthen the network lifespan by minimizing the energy consumption of the system. The energy loss in the network was occurs due to the data transmission and reception process which happens based on the distance and the packet size. For the selection of the finest cluster head and the cluster head quantity, this YSGA has been introduced with the routing protocol. By using the YSGA technique, the energy consumption was reduced. Hence the network lifespan also improved. However, this developed protocol was able to execute in only off-line mode.

\section{Proposed method}

Clustering methods have various significant advantages. Clustering provides energy efficiency, scalability to decrease the energy utilization of SNs in HWSNs. Clustering reduces network overhead and traffic. Clustering prevents redundant messages from being exchanged during the communication. In clustering, CHs perform data compression and data aggregation task, which results in reduced energy consumption in the network. Also, the transmission range among SNs reduces in clustering mechanism. In the clustering mechanism, only $\mathrm{CHs}$ are having high-range data transmission. In the clustering mechanism, CHs play a major role, and therefore their selection is one of the most critical problems in the clustering process. The traditional optimization algorithms are not capable enough to provide the appropriate solution in a specific time. Metaheuristic methods are mainly used for these optimization problems to provide the best solution.

The proposed hybrid AHCS-GWO algorithm provides the best solution by minimizing all objective function. The fitness function for this is the sum of all objective functions with seven weight constants. The fitness function, which is defined in the proposed method, is described as follows, in order to enhance the local search ability and optimization speed of the algorithm, this paper introduces the mutation operation operator, and presents a new evolution strategy of the hybrid cuckoo search algorithm.

\subsection{Adaptive hybrid cuckoo search}

AHCS method consists of two key constraints, one is a direct exploration that depends on Levy flights and the second is a random exploration that depends on the probability of a host bird to determine an alien egg present in the nest. 
Unhealthy eggs are recognized by the host bird through a probability $P a \in[0,1]$ and its constraints are nested number $(n)$, discovery rate $(p a)$, and levy coefficient $\lambda$.

The cuckoo indiscriminately selects the position of the nest $\left(X_{p q}^{g e n+1}\right)$ to place eggs via Eqs. (1) and (2).

$$
\begin{gathered}
X_{p q}^{g e n+1}=X_{p q}^{g e n}+S_{p q} \times \operatorname{Levy}(\lambda) \times \alpha \\
\operatorname{Levy}(\lambda)=\left|\frac{\Gamma(1+\lambda) \times \sin \left(\frac{\pi \times \lambda}{2}\right)}{\Gamma\left(\frac{1+\lambda}{2}\right) \times \lambda \times S^{(\lambda-1) / 2}}\right|^{1 / \lambda}
\end{gathered}
$$

where $\lambda$ is constant $(1<\lambda \leq 3)$; random number generated between $[-1,1]$ is expressed as $\alpha$; gamma function is stated as $\Gamma$; step size is taken as $\mathrm{S}>0$. The optimal step size is attained by Eq. (3).

$$
S_{p q}=X_{p q}^{g e n}-X_{f q}^{g e n}
$$

where $p, f \epsilon\{1,2, \ldots ., m\}$ and $\mathrm{q} \epsilon\{1,2, \ldots, D\}$ are randomly chosen indexes, $f$ is chosen randomly but its value must be different from $p$.

When the host bird finds the cuckoo egg and selects the particular egg value with probability using Eq. (4).

$$
\operatorname{pro}_{q}=\left(\frac{0.9 \times f i t_{q}}{\max (f i t)}\right)+0.1
$$

where fitness value is specified as $f i t_{q} ; q$ is specified as the proportionality index present in the nest position $q$. Here, the constant values of $0.9 \&$ 0.1 are considered to convert the multiple objective values into single objective. Because each objective value has different range value.

If the host bird recognizes the cuckoo egg, it will toss the egg outside and establish a new nest by using Eq. (5). Or else the egg will propagate and is prosperous for the succeeding group.

$$
\operatorname{nest}_{q}=X_{q, \min }+\operatorname{rand}(0,1) \times\left(X_{q, \max }-X_{q, \min }\right)
$$

When the improved CS performs a local search, the algorithm may still have a locally optimal situation. In order to reduce the probability that CS falls into local optimum, this paper proposes a new evolutionary strategy. The evolutionary strategy of the adaptive hybrid cuckoo search is as follows:

(1) Timing begins, initialize the relevant parameters of AHCS, such as the population size $N$, disturbed iteration threshold $T_{0}$, the maximum runtime of the algorithm maxruntime and so on.

(2) The initial population is generated as follows:

$$
X_{p q}^{g e n}=a+\operatorname{rand}(1, D) *(b-a)
$$

where $X_{p q}^{g e n}$ is an individual in the randomly generated initial population, $b$ is the upper bound vector of the variable, $a$ is the lower bound vector of the variable, $D$ is the dimension of the variable, rand $(1, D)$ is a random uniform vector in $[0,1]$, and ".* " shows the dot product of two vectors.

(3) Calculate the fitness values of all nest positions in the population, and sort the fitness from small to large, and record the best nest position and its fitness.

(4) For each nest, according to Eq. (6), the positional update of the Lévy flight is performed on the AHCS. After that, the fitness value of the nest position after the position update is calculated, and compared with the fitness of the bird's nest position before the update, the better bird's nest position and its fitness value are retained.

(5) Record the running time of the algorithm runtime, if the iteration stop condition is satisfied, the optimal solution and the optimal value are output; if not, then return to (4).

\subsection{Grey wolf optimization}

GWO is generally inspired by leadership and hunting behavior of the grey wolves. The grey wolves are categorized into four levels based on the social dominant hierarchy such as alpha wolf $(\alpha)$, beta wolf $(\beta)$, delta wolf $(\delta)$ and omega wolf $(\omega)$. The GWO is generally depends on the following assumptions: 1) The $\alpha, \beta$ and $\delta$ are denoted as optimum, 2nd optimum and 3rd optimum solution respectively. 2) The remaining level is supposed to be omega wolf $(\omega)$. 3) The three wolves such as alpha wolf, beta wolf and delta wolf are considered as optimum solution that has better information about the potential location of prey. The information about prey of those three wolves are better than the omega wolf. 4) the omega wolf follows the three best wolves. The global best position (gbest) from the AHCS is considered as location vector of prey. The process of GWO are given as follows:

\section{a. Encircling prey}

The Eq. (7) defines the encircling behavior of grey wolves. 


$$
Y^{t+1}=Y_{p}^{t}-B^{t} \times\left|D^{t} \times Y_{p}^{t}-Y^{t}\right|
$$

where, the prey's location vector is represented as $Y_{p}^{t}$; the coefficient vectors are $B^{t}$ and $D^{t}$; the grey wolf's position vector is $Y^{t}$. The Eqs. (8) and (9) are represented the coefficient vector of $B^{t}$ and $D^{t}$ respectively.

$$
\begin{gathered}
B^{t}=2 b^{t} \text { rand }_{1}-b^{t} \\
D^{t}=2 \text { rand }_{2}
\end{gathered}
$$

where, the exploration rate is specified as $b^{t}$. The rand $_{1}$ and rand $_{2}$ are represented the random vectors among 0 and 1 . The exploration rate is linearly minimized from 2 to 0 over the number of iterations. The exploration rate is specified in the Eq. (10).

$$
b_{j}^{t}=2-\frac{2 t}{N_{\max }}
$$

where, $N_{\max }$ specifies the maximum amount of iterations.

\section{b. Hunting}

The hunting process of the grey wolves are handled by the alpha wolf. The beta and delta wolf may also participate as guide for hunting at sometimes. Besides, it is very difficult to obtain the prey location in search space. The three wolves such as alpha wolf, beta wolf and delta wolf has better information about the potential location of prey. These prey locations are used to process the hunting behavior of grey wolves. The Eqs. (11) to (13) are used to stimulate the hunting process of the GWO.

$$
\begin{aligned}
& Y_{1}=Y_{\alpha}^{t}-B_{1}^{t} \times\left|D_{1}^{t} \times Y_{\alpha}^{t}-Y^{t}\right| \\
& Y_{2}=Y_{\beta}^{t}-B_{2}^{t} \times\left|D_{2}^{t} \times Y_{\beta}^{t}-Y^{t}\right|
\end{aligned}
$$

$$
Y_{3}=Y_{\delta}^{t}-B_{3}^{t} \times\left|D_{3}^{t} \times Y_{\delta}^{t}-Y^{t}\right|
$$

where, the $Y_{\alpha}^{t}, Y_{\beta}^{t}$ and $Y_{\delta}^{t}$ are the position of the alpha wolf, beta wolf and delta wolf respectively. The average state of the position obtained from the alpha wolf, beta wolf and delta wolf are given in Eq. (14). This average position gives the optimum position of the grey wolf.

$$
Y^{t+1}=\frac{Y_{1}+Y_{2}+Y_{3}}{3}
$$

\subsubsection{Fitness function derivation}

The fitness function used of the AHCS-GWO method is derived in this section. The expression for fitness function is given in the following Eq. (15).

$$
\text { Mean }=\frac{\sum_{i=1}^{R} f_{i}}{R}
$$

where $f_{i}$ is defined as the best fitness value that the algorithm achieved in the $i$-th run.

\subsection{AHCS-GWO based CH selection}

Mobile sensors have been intensively researched to improve a WSN's topology. Moving sensors to approximate the event distribution, while maintaining complete coverage of the sensing field. The solution must dispatch one Mobile Sensor (MS) into one Event Location (EL) in each odd round and then dispatch the same mobile sensor back to its original location in the subsequent even round.

The proposed approach consists of mobile node selection and routing algorithms. The first algorithm effectively chooses the near optimal set of sensor nodes as CHs among all sensor nodes. Afterward, derived potential function is used to assign non- $\mathrm{CH}$ nodes to the $\mathrm{CH}$ nodes. Next, the routing algorithm is proposed to find the near optimal path from every mobile node to the event location. The idea is to

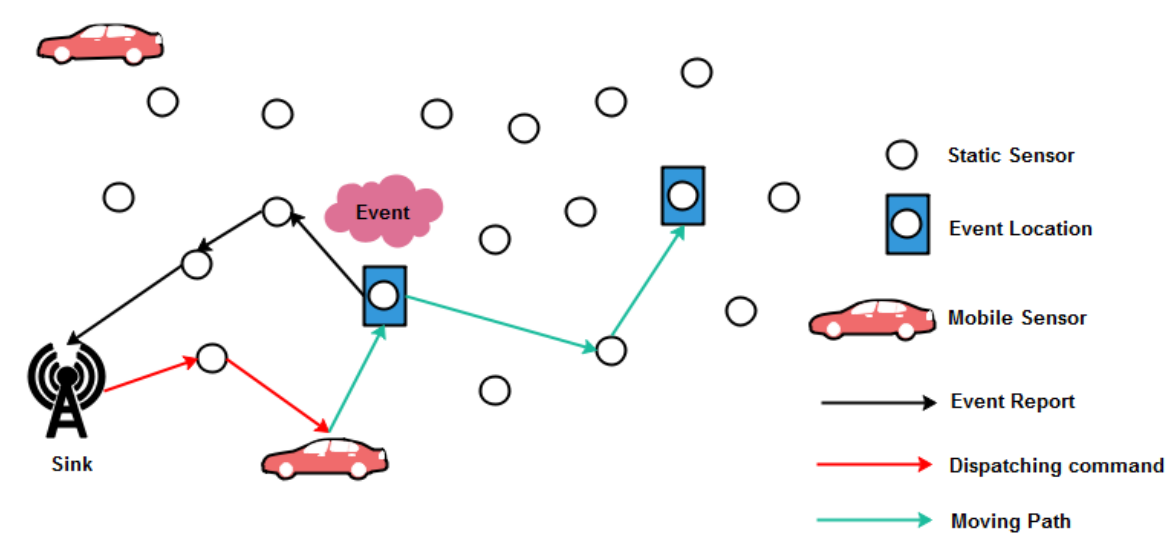

Figure. 1 Sensor dispatch in Hybrid WSN 
minimize their moving energy while keeping their energy consumption balanced after each round. Without loss of generality, delete those mobile sensors that do not have sufficient energy to reach any location. Fig 1 shows the dispatch between the mobile sensor and event location along with the moving path in a hybrid wireless sensor network.

\subsubsection{Representation and initialization}

In the $\mathrm{CH}$ selection process, the dimension of population is equal to the number of $\mathrm{CHs}$ in the network. Let $P_{i}=P_{i}^{1}, P_{i}^{2}, \ldots P_{i}^{m}$ be $i^{\text {th }}$ population, where every position $P_{i}^{d}=\left(x_{i}^{d}, y_{i}^{d}\right)$ represents node_id of a mobile node and is mapped with its $2 \mathrm{D}$ co-ordinates in the network.

\subsubsection{Derivation of fitness function}

Its aim is to select near optimal set of sensor nodes as CHs. To achieve this objective, a fitness function is formulated using intra-cluster distance, residual energy, distance from mobile node to the event location.

\section{(a) Residual energy of cluster head:}

In data transmission phase, mobile node receives data from non-CH nodes then transmits to the event location after aggregation. In order to perform these tasks, mobile node requires more residual energy. Therefore, sensor node with higher residual energy is a more preferable choice as a mobile node. So, our first objective with respect to residual energy is $f_{1}$ which can be minimized as follows:

$$
\text { Minimize } f_{1}=\sum_{i=1}^{m} \frac{1}{E_{C H i}}
$$

\section{(b) Intra-cluster distance:}

It is the euclidean distance between non-mobile sensor nodes to their respective mobile node. In the data transmission process, a sensor node consumes more energy than other tasks as mentioned. If transmission distance is minimum, then less energy is required to process the data. So, our second objective in terms of intra-cluster distance is $f_{2}$ which can be minimized as follows:

$$
\text { Minimize } f_{2}=\sum_{j=1}^{m}\left(\sum_{i=1}^{I_{j}} \operatorname{dis}\left(s_{i}, C H_{j}\right) / I_{j}\right)
$$

\section{(c) Distance between mobile node to event location:}

It is the euclidean distance between each Mobile Node to the Event Location (EL). As mentioned earlier, energy consumption of a sensor node depends on the transmission distance. If event location is located far away from the mobile node, then it requires large amount of energy to accomplish its task. Rapid fall in the power of mobile node threatens the performance of the network. Therefore, mobile node with minimum euclidean distance from EL is a more preferable choice. So, our third objective is $f_{3}$ which can be minimized as follows:

$$
\text { Minimize } f_{3}=\sum_{i=1}^{m}\left(\operatorname{dis}\left(C_{j}, E L\right)\right)
$$

\section{(d) Node degree:}

It is the number of non-CH members belongs to its respective mobile node. If mobile node has lesser number of members, then it sustains for longer duration. Therefore, mobile node with least node degree is preferred. So, our fourth objective is $f_{4}$ which can be minimized as follows

$$
\text { Minimize } f_{4}=\sum_{i=1}^{m} I_{i}
$$

All the mentioned above objectives are nonconflicting in nature, thus instead of minimizing them separately, then convert these objectives into a single objective function using weighted-sum approach. Moreover, that all objectives have different range of values. Therefore, normalization function is applied to each objective and here $\alpha_{1}, \alpha_{2}$, $\alpha_{3}, \alpha_{4}$ are weights assigned to each objective.

$$
F(x)=\frac{f_{i}-f_{\min }}{f_{\max }-f_{\min }}
$$

where $f_{i}$ is the value of the function, $f_{\min }$ is minimum value, $f_{\max }$ is maximum value and $F(x)$ is the normalized value between 0 and 1 .

$$
\text { Minimum fitness }=\alpha_{1} f_{1}+\alpha_{2} f_{2}+\alpha_{3} f_{3}+\alpha_{4} f_{4}
$$

$$
\text { where } \sum_{\mathrm{i}=1}^{4} \alpha_{i}=1 \text {; and } \alpha_{i} \in(0,1)
$$

\subsection{AHCSGWO-based routing}

\subsubsection{Representation and initialization}

In the routing process, dimension of each population is equal to the number of mobile node $(m)$. Let $P_{i}=P_{i}^{1}, P_{i}^{2}, \ldots P_{i}^{m}$ be $i^{t h}$ population, where each dimension of population such as $P_{i}^{1}=$ $(0,1)$, is randomly initialized by a real number between 0 and 1 . Afterward, a novel mapping 
function is applied to calculate the next-hop toward the EL.

\subsubsection{Derivation of fitness function}

The main objective is to select near optimal path from each mobile node to the event location. To accomplish this task, a fitness function is formulated using residual energy, distance and node degree.

\section{(a) Residual energy of next-hop node:}

In the data transmission process, $\mathrm{CH}$ selects nearer next-hop to proceed its data toward the EL but selected next-hop also have its own task, i.e., to proceed data from its members after aggregation. Therefore, next-hop with more residual energy is a more preferable choice. So, our first objective associated with residual energy is $f_{1}$ which can be maximized as follows:

\section{Objective 1}

$$
\text { Maximize } f_{1}=\sum_{i=1}^{m} E_{C H i}
$$

\section{(b) Distance:}

It is the Euclidean distance between the mobile node to the next-hop and from there to the event location. As mentioned, energy consumption is determined by transmission distance. If it is minimum, then it expenses less amount of energy. Therefore, second objective is to minimize both the distances together, i.e., mobile node to next-hop and next hop to the event location. It will increase the life of the network. So, second objective associated with distances is $f_{2}$ which can be maximized as follows:

\section{Objective 2}

$$
\text { Maximize } f_{2}=\frac{1}{\sum_{i=1}^{m} \operatorname{dis}\left(C H_{i}, N H\right)+\operatorname{dis}(N H, E L)}
$$

\section{(c) Node degree}

It is the number of non- $\mathrm{CH}$ member belonging to a respective $\mathrm{CH}$. In next-hop selection, one important factor is node degree. If next-hop selected with less node degree, then it receives less data from its members and perform task for long time. Therefore, next-hop with low node degree is a more preferable choice. So, third objective associated with node degree is $f_{3}$ which can be maximized as follows

\section{Objective 3}

$$
\text { Maximize } f_{3}=\frac{1}{\sum_{i=1}^{m} I_{i}}
$$

All aforementioned objectives have different range values, in order to convert into same range normalization function is applied as mentioned earlier. Here $\emptyset_{1}, \emptyset_{2}$ and $\emptyset_{3}$ are weights associated with each objective.

$$
\text { Maximize Fitness }=\emptyset_{1}\left(f_{1}\right)+\emptyset_{2}\left(f_{2}\right)+\emptyset_{3}\left(f_{3}\right)
$$

where $\sum_{i=1}^{3} \emptyset_{i}=1, \emptyset_{i} \in(0,1)$

The flowchart for the AHCS-GWO method is shown in below Fig 2. The steps for the flowchart are mentioned as follows:

- Initially, the sensor nodes are randomly deployed in the interested area. The mobile node and the event location are fixed depends on the location of the sensor nodes.

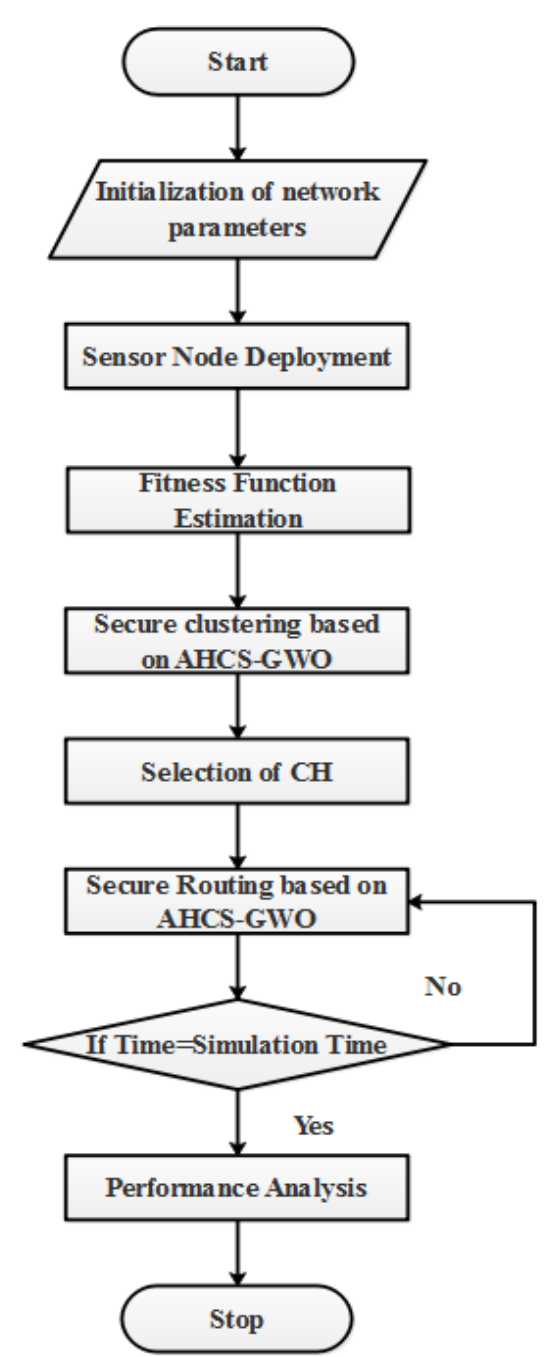

Figure. 2 Flowchart for the proposed AHCS-GWO 
- Clustering algorithm is developed in HWSN to divide the network into clusters. In this research, AHCS-GWO is implemented to cluster the network. Then the $\mathrm{CH}$ from each cluster is selected based on residual energy, distance to the neighbors, distance to the event location, etc.

- The optimal route among the mobile node to event location is obtained by using the routing protocol. Here, the routing protocol is developed by using the proposed AHCS-GWO algorithm.

- From the routing an optimized node is selected to generate the route from mobile node to the event location.

- After creating the path from source to destination, the source node transmits the data to the destination.

- This proposed AHCS-GWO method predicts the optimal path by considering three objective functions such as residual energy of the nodes, distance between the mobile node to the event location and the number of hops.

- The residual energy of the nodes is monitored frequently by the event location. The reclustering/rerouting is performed often to avoid packet loss in the network.

\section{Result and discussion}

In this research, Network simulator - 2 has been used as the simulator for implementing and testing the proposed energy-efficient routing algorithm. The objective of this research article is to minimize the energy consumption of each node in an intelligent manner as dictated by the routing scheme. A clusterbased routing scheme is employed with optimal cluster head selection done by the cuckoo search algorithm. The input to the proposed algorithm to perform optimization is composed of a quadruple namely \{energy consumption, hop distance, packet size, node location $\}$. Based on these attributes, it chooses the optimal $\mathrm{CH}$ which initiates and coordinates the complete transfer of information from source to destination with least energy consumption which could be observed in the results shown below.

Following the setting of configuration parameters as listed in Table 1. The proposed energy-aware routing scheme has been successfully implemented and tested with Low Energy Adaptive Clustering Hierarchy (LEACH) [14], PowerEfficient Gathering in Sensor Information Systems (PEGASIS) [14], threshold-sensitive energy efficient sensor network (TEEN) [14] routing algorithm, EMEER [14] and YSGA [17] as well as superior performance observed in terms of packet delivery ratio, energy consumption, average energy consumption of each node and throughput.

Computation time analysis for varying node count is shown in Fig 3 in which AHCS-GWO method achieves less response time than the PEGASIS [14], LEACH [14], TEEN [14], EMEER [14] and YSGA [17].

Table 1. Simulation specification

\begin{tabular}{|c|c|}
\hline Parameter & Value \\
\hline Simulation time & $180 \mathrm{~s}$ \\
\hline Network size & $100091000 \mathrm{~m}$ \\
\hline Number of sensor nodes & $100-200$ \\
\hline Radio propagation range & $250 \mathrm{~m}$ \\
\hline Channel capacity & $2 \mathrm{M}$ bits/s \\
\hline Physical layer & IEEE $802.11 \mathrm{~b}$ DCF \\
\hline Data packet size & 1000 bytes \\
\hline
\end{tabular}

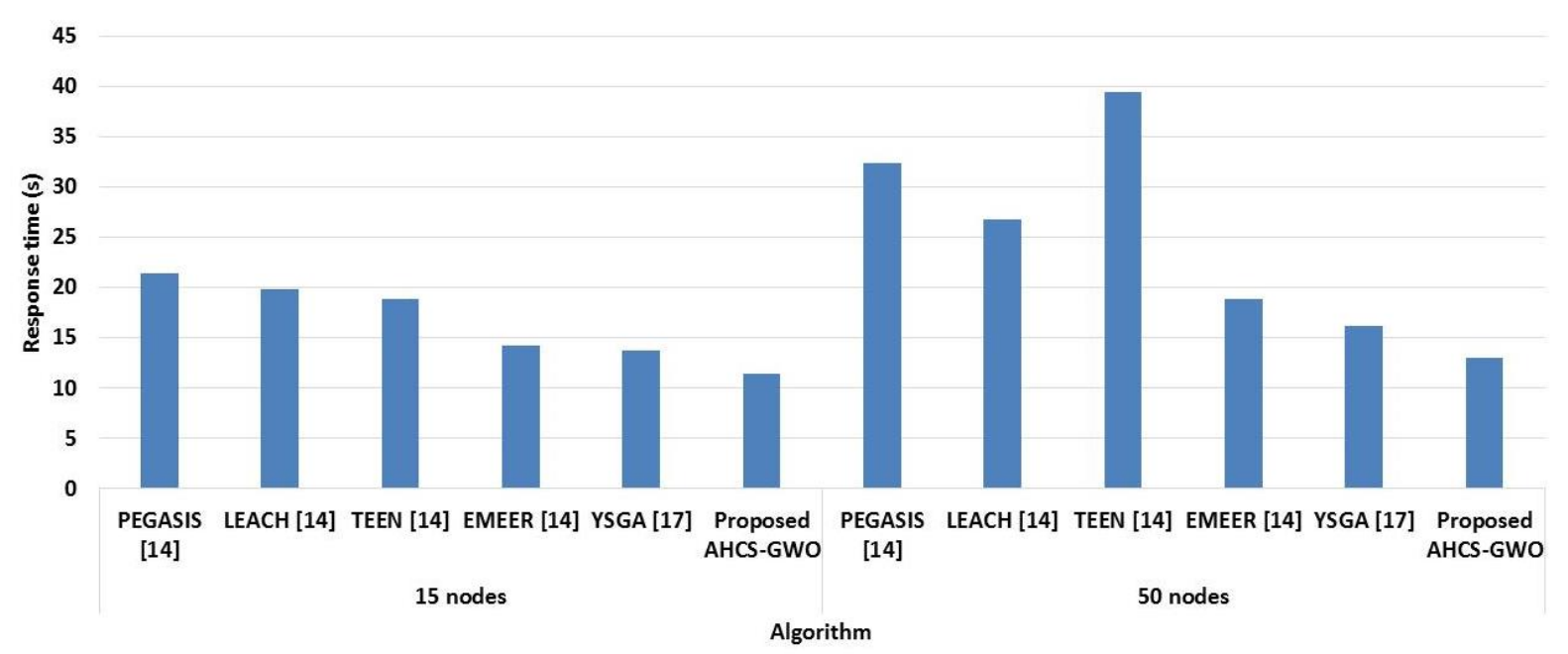

Figure. 3 Response Time of Proposed AHCS-GWO 
The outcome of the projected AHCS-GWO routing procedure is enumerated in terms of Packet Drop Ratio (PDR), end-to-end delay, residual energy and energy consumption of the nodes. Fig 3 illustrates the performance of the proposed AHCSGWO algorithm with PEGASIS [14], LEACH [14], TEEN [14], EMEER [14] and YSGA [17]. It could be seen that the end-to-end delay in the proposed work is drastically reduced up to 7.729 for a maximum node count of 200 nodes as well as it is reduced up to 4.092 when the number of nodes is 100. The response of computation time involved in routing from source to destination is listed in Fig 3. The analyses have been done by varying the node count for 15 and 50 nodes, and it could be observed in each case that the proposed AHCS-GWO routing scheme exhibits reduced computation time by up to $37.13 \%$ for 50 nodes, when compared to the EMEER [14].

The following performance metrics are qualifying the presentation of the projected AHCSGWO is the Packet Drop Ratio (PDR) that is perceived in the graph represented in Fig. 4. It might be evidently perceived that the projected AHCSGWO method overcomes the traditional clusterdependent procedures like PEGASIS [14], LEACH [14], TEEN [14], EMEER [14] and YSGA [17]. Those techniques confirming a maximum transmission of data from mobile node to event location that is contemplative from the condensed PDR investigation. The ratio of PDR is decreased up to $9.71 \%$ and $5.49 \%$ for AHCS-GWO method with 200 nodes, when compared to the EMEER [14] and YSGA [17] respectively.

Fig. 5 depicts the performance analysis of end-toend delay when compared against the existing techniques. The proposed AHCS-GWO gives superior performance than the PEGASIS [14], LEACH [14], TEEN [14], EMEER [14] and YSGA [17].

Fig. 6 illustrates the residual energy analysis in a similar manner. Fig 6 depicts the energy consumption analysis measured in Joules with increasing number of node counts.

Fig. 7 displays the comparison of the energy consumption values for PEGASIS [14], LEACH [14], TEEN [14], EMEER [14] and YSGA [17] for accomplishing the event location from the mobile node. At every single node, the projected AHCSGWO algorithm reached the event location with a smaller amount consumption of energy while associated with the existing algortihm. From the Fig 7, it clearly indicates that the proposed AHCS-GWO consumes less power for reaching the event location when compared with the existing PEGASIS [14],
LEACH [14], TEEN [14], EMEER [14] and YSGA [17].

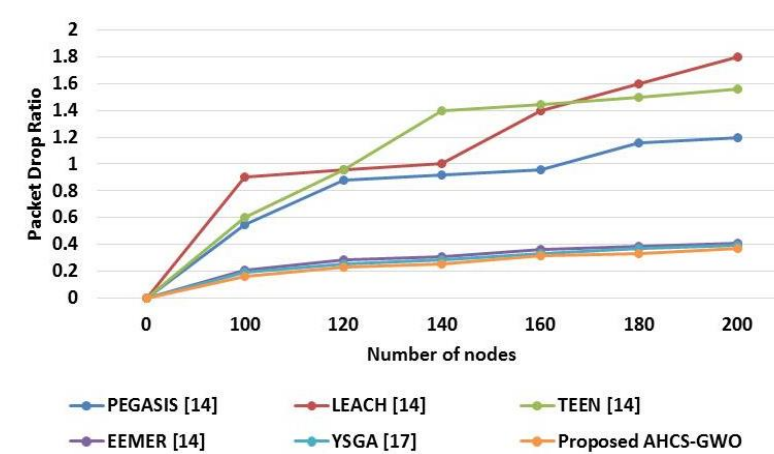

Figure. 4 Performance of packet drop ratio for Proposed AHCS-GWO

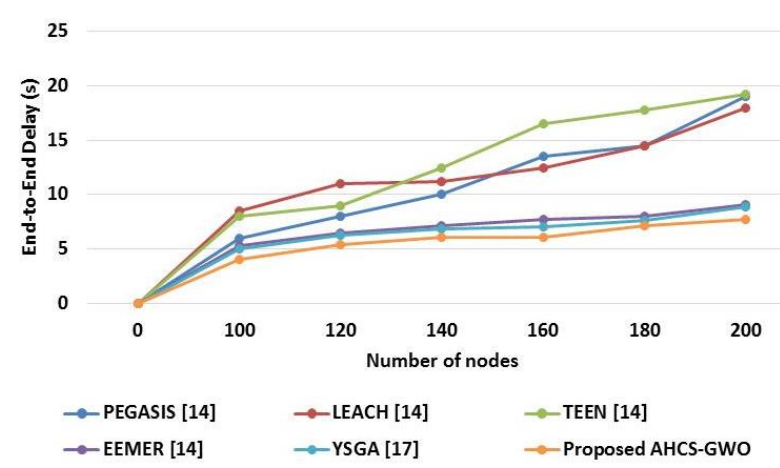

Figure. 5 Performance of end-to-end delay for Proposed AHCS-GWO

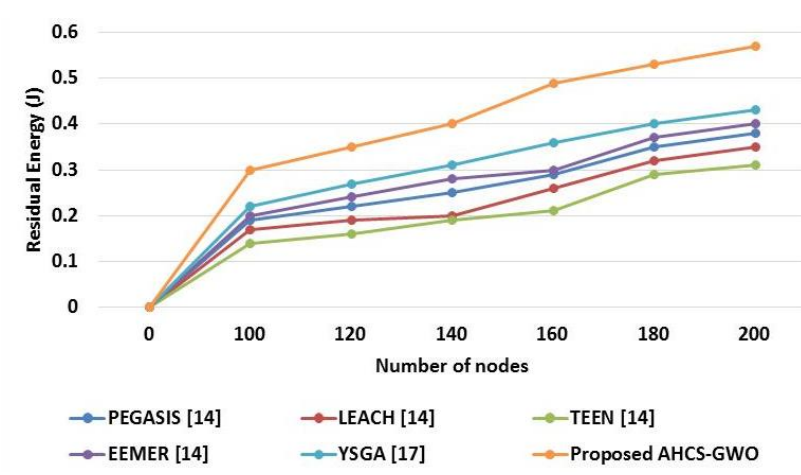

Figure. 6 Residual energy analysis in the proposed AHCS-GWO

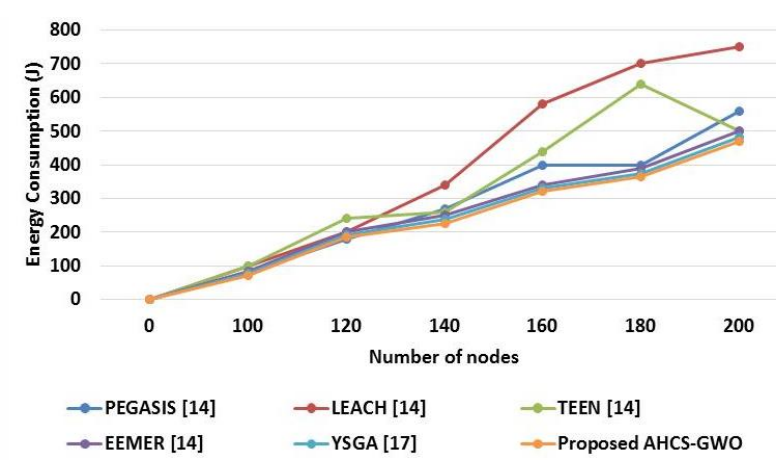

Figure. 7 Energy consumption analysis of the proposed AHCS-GWO 


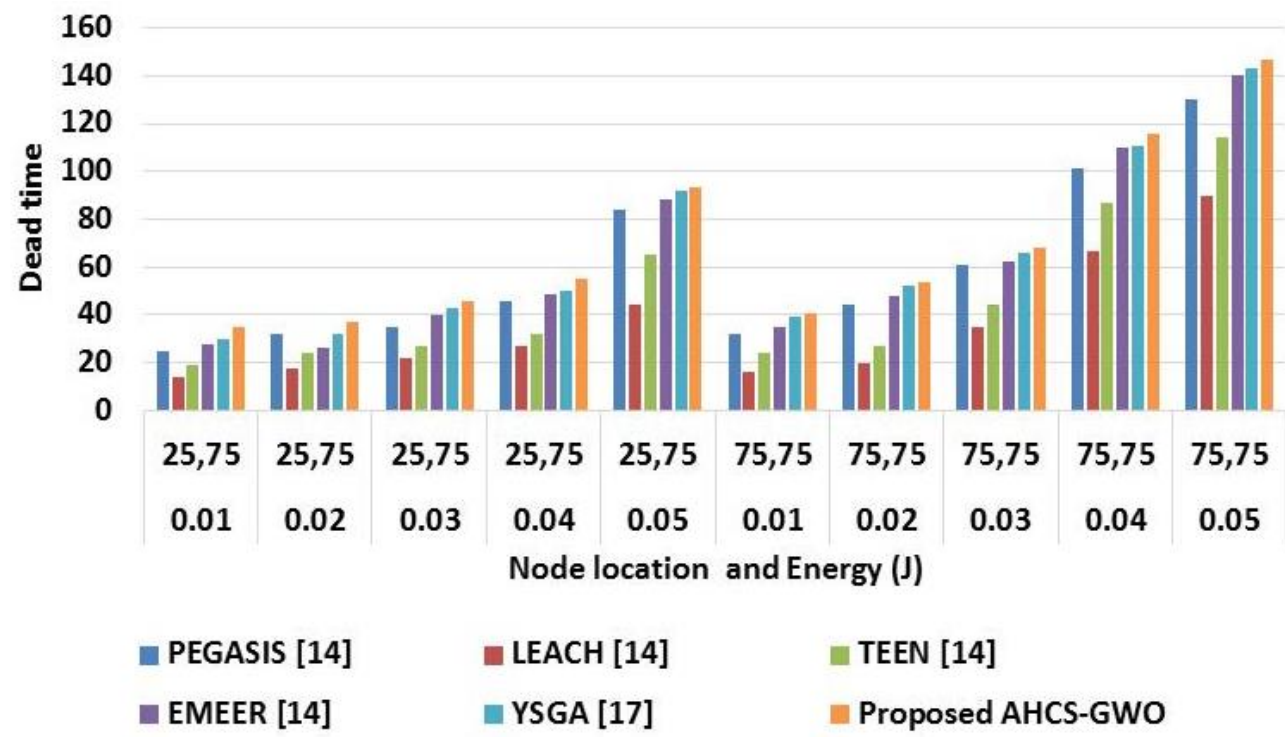

Figure. 8 Performance analysis of Dead Time Analysis

Table 2. Performance analysis of dead time

\begin{tabular}{|c|c|c|c|c|c|c|c|}
\hline $\begin{array}{c}\text { Energy } \\
(\mathbf{J})\end{array}$ & $\begin{array}{c}\text { Node } \\
\text { location }\end{array}$ & $\begin{array}{c}\text { PEGASIS } \\
{[\mathbf{1 4}]}\end{array}$ & $\begin{array}{c}\text { LEACH } \\
{[\mathbf{1 4}]}\end{array}$ & $\begin{array}{c}\text { TEEN } \\
{[\mathbf{1 4}]}\end{array}$ & $\begin{array}{c}\text { EMEER } \\
{[\mathbf{1 4}]}\end{array}$ & $\begin{array}{c}\text { YSGA } \\
{[\mathbf{1 7}]}\end{array}$ & $\begin{array}{c}\text { Proposed AHCS- } \\
\text { GWO }\end{array}$ \\
\hline 0.01 & 25,75 & 25 & 14 & 19 & 28 & 30 & 35 \\
\hline 0.02 & 25,75 & 32 & 18 & 24 & 26 & 32 & 37 \\
\hline 0.03 & 25,75 & 35 & 22 & 27 & 40 & 43 & 46 \\
\hline 0.04 & 25,75 & 46 & 27 & 32 & 49 & 50 & 55 \\
\hline 0.05 & 25,75 & 84 & 44 & 65 & 88 & 92 & 93 \\
\hline 0.01 & 75,75 & 32 & 16 & 24 & 35 & 39 & 41 \\
\hline 0.02 & 75,75 & 44 & 20 & 27 & 48 & 52 & 54 \\
\hline 0.03 & 75,75 & 61 & 35 & 44 & 62 & 66 & 68 \\
\hline 0.04 & 75,75 & 101 & 67 & 87 & 110 & 111 & 116 \\
\hline 0.05 & 75,75 & 130 & 90 & 114 & 140 & 143 & 147 \\
\hline
\end{tabular}

\subsection{Comparative analysis}

Dead time analysis is presented in Table 2 and its first node are compared against the PEGASIS [14], LEACH [14], TEEN [14], EMEER [14] and YSGA [17]. Fig 8 illustrates the dead time analysis of existing and proposed AHCS-GWO algorithm. From the Fig 8, it clearly indicates that proposed AHCS-GWO algorithm displays determined nodes alive time earlier it expires because of exhausted energy. The calculation time is completed by deliberating the communication route from mobile node to event location designated through position which organizes 25-75 nodes denoting the source node and end point, correspondingly.

From the performance analysis, it is concluded that the AHCS-GWO provides better performance than the PEGASIS [14], LEACH [14], TEEN [14], EMEER [14] and YSGA [17]. The AHCS-GWO provides better performance due to its optimal $\mathrm{CH}$ selection and routing path generation. The energy consumption of the AHCS-GWO method is minimized mainly by considering the distance between the static and mobile sensors of the HWSN. Accordingly, the dead time of the AHCS-GWO method is increased in the HWSN. Moreover, the node and link failure are avoided in the routing due to an appropriate fitness function. This results in lesser packet drop during the communication. Therefore the energy consumption of the AHCSGWO method is minimized while minimizing the packet drop.

\section{Conclusion}

In this research study, a hybrid WSN has been taken up for exploration and an energy-efficient routing procedure established on an evolutionary optimization process has been projected. Here, Adaptive Hybrid Cuckoo Search with Grey Wolf Optimization is proposed for attaining the event location through a less energy consumption. This algorithm is hybrid in nature connecting the traditional clustering to divide the specified system 
into clusters based on resemblance and energy-level structures monitored by an optimum cluster head by means of the nature-inspired cuckoo search procedure. The suggested combination of AHCSGWO method is executed for a system dimension extending from 100 to 200, and its energy efficiency would reveal the lifespan of the complete network which is investigated in terms of energy consumption, residual energy, end-to-end delay and Packet Drop Ratio (PDR). From the results, it clearly indicates in all the cases, the proposed AHCS-GWO algorithm outperforms than the PEGASIS, LEACH, TEEN, EMEER and YSGA. The residual energy of the AHCS-GWO method for 200 nodes is $0.57 \mathrm{~J}$ which is high when compared to the PEGASIS, LEACH, TEEN, EMEER and YSGA methods. In future, the performances of the HWSN can be increased by using the novel optimization algorithms.

\section{Conflicts of Interest}

The authors declare no conflict of interest.

\section{Author Contributions}

The paper conceptualization, methodology, software, validation, formal analysis, investigation, resources, data curation, writing-original draft preparation, writing-review and editing, visualization, have been done by $1^{\text {st }}$ author. The supervision and project administration, have been done by $2^{\text {nd }}$ author.

\section{References}

[1] J. Wang, Y. Gao, W. Liu, A. K. Sangaiah, and H. J. Kim, "Energy efficient routing algorithm with mobile sink support for wireless sensor networks", Sensors, Vol. 19, No. 7, pp. 1494, 2019.

[2] Y. C. Wang, W. C. Peng, and Y. C. Tseng, "Energy-balanced dispatch of mobile sensors in a hybrid wireless sensor network", IEEE Transactions on Parallel and Distributed Systems, Vol. 21, No. 12, pp. 1836-1850, 2010.

[3] G. K. Shwetha, S. Behera, and J. Mungara, "Energy-balanced dispatch of mobile sensors in hybrid wireless sensor network with obstacles", IOSR Journal of Computer Engineering, Vol. 2, No. 1, pp. 47-51, 2012.

[4] S. Rani, J. Malhotra, R. Talwar, "Energy efficient chain based cooperative routing protocol for WSN", Applied Soft Computing, Vol. 35, pp. 386-397, 2015.
[5] R. Singh, and A. K. Verma, "Energy efficient cross layer based adaptive threshold routing protocol for WSN", AEU-International Journal of Electronics and Communications, Vol. 72, pp. 166-173, 2017.

[6] M. Shafiq, H. Ashraf, A. Ullah, and S. Tahira, "Systematic Literature Review on Energy Efficient Routing Schemes in WSN-A Survey", Mobile Networks and Applications, pp. 1-14, 2020.

[7] M. Elhoseny, X. Yuan, H. K. E. Minir, and A. M. Riad, "An energy efficient encryption method for secure dynamic WSN", Security and Communication Networks, Vol. 9, No. 13, pp. 2024-2031, 2016.

[8] G. S. Arumugam and T. Ponnuchamy, "EELEACH: development of energy-efficient LEACH Protocol for data gathering in WSN", EURASIP Journal on Wireless Communications and Networking, Vol. 2015, No. 1, pp. 1-9, 2015.

[9] A. Shukla, and S. Tripathi, "A multi-tier based clustering framework for scalable and energy efficient WSN-assisted IoT network", Wireless Networks, pp. 1-23, 2020.

[10] A. Shukla and S. Tripathi, "An effective relay node selection technique for energy efficient wsn-assisted iot", Wireless Personal Communications, Vol. 112, No. 4, pp. 26112641, 2020.

[11] Z. Wang, H. Ding, B. Li, L. Bao, and Z. Yang, "An energy efficient routing protocol based on improved artificial bee colony algorithm for wireless sensor networks", IEEE Access, Vol. 8, pp. 133577-133596, 2020.

[12] T. A. Alghamdi, "Route optimization to improve QoS in multi-hop wireless sensor networks", Wireless Networks, pp. 1-7, 2020.

[13] A. Nayyar and R. Singh, "IEEMARP-a novel energy efficient multipath routing protocol based on ant Colony optimization (ACO) for dynamic sensor networks", Multimedia Tools and Applications, pp. 1-32, 2019.

[14] M. Ezhilarasi and V. Krishnaveni, "An evolutionary multipath energy-efficient routing protocol (EMEER) for network lifetime enhancement in wireless sensor networks", Soft Computing, Vol. 23, No. 18, pp. 8367-8377, 2019.

[15] X. Li, B. Keegan, F. Mtenzi, T. Weise, and M. Tan, "Energy-efficient load balancing ant based routing algorithm for wireless sensor networks", IEEE Access, Vol. 7, pp. 113182-113196, 2019.

[16] C. Xu, Z. Xiong, G. Zhao, and S. Yu, "An energy-efficient region source routing protocol 
for lifetime maximization in WSN", IEEE Access, Vol. 7, pp. 135277-135289, 2019.

[17] A. Rodríguez, C. D. V. Soto, and R. Velázquez, "Energy-Efficient Clustering Routing Protocol for Wireless Sensor Networks Based on Yellow Saddle Goatfish Algorithm", Mathematics, Vol. 8, No. 9, pp. 1515, 2020. 